\title{
Thermal Crosstalk of Integrated DFB Laser-EA Modulators
}

\author{
Kayastha Madhusudan Non-member (Chubu University) \\ Koichi Wakita Member (Chubu University) \\ Kiyohide Baba Non-member (Chubu University)
}

Keywords : integrated, DFB laser, thermal

\section{Introduction}

Integrated distributed feedback (DFB) laser diodes and electroabsorption modulators (EAM) are attractive light source for high-bit-rate and long-distance optical fiber transmission systems because of their low or even negative chirp parameters, small size and low driving voltages compared with other modulator types. However, they show a red shift of emission wavelength with applied bias voltage. This leads to increase in an adiabatic chirp parameter that is usually zero to decoupled devices.

The thermal crosstalk model has been reported for identical InGaAs/InAlAs MQW double-stack active layer ${ }^{(1)}$, which is of very special structure and not applied for practically used InGaAsP/InGaAsP MQWs. In this paper, we try to take measurement of InGaAsP /InGaAsP MQW integrated device and to make corresponding simulation that the phenomenon is identified to be caused by thermal coupling between the sections.

\section{Device and Measurement Setup}

The device contact setup is displayed in Fig. 1. A detailed report on the fabrication steps is given $\mathrm{in}^{(2)}$. The active area consists of an InGaAsP/InGaAsP multiple quantum well (MQW) structure on $\mathrm{n}$-InP substrate. This is able to deliver high gain for forward current injection and high absorption swing for reverse voltage at $1542 \mathrm{~nm}$ operation wavelength. Two different contacts that are induced as DFB and EAM are aligned along a stripe waveguide buried in Fe-doped InP to reduce electric capacitance. These two sections are butt-joint configuration with efficiency of $95 \%$.

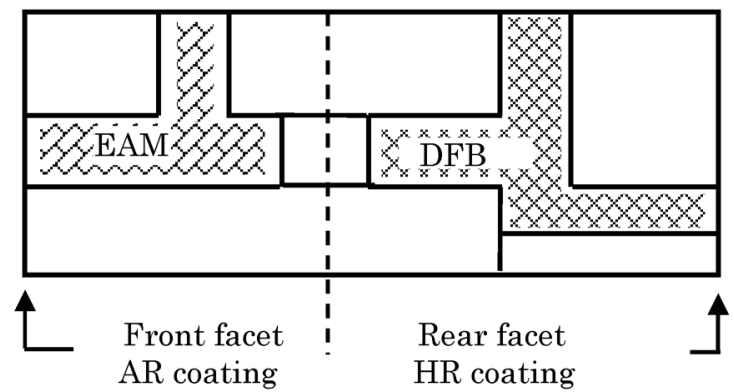

Fig. 1. Top view of the contact design of the device

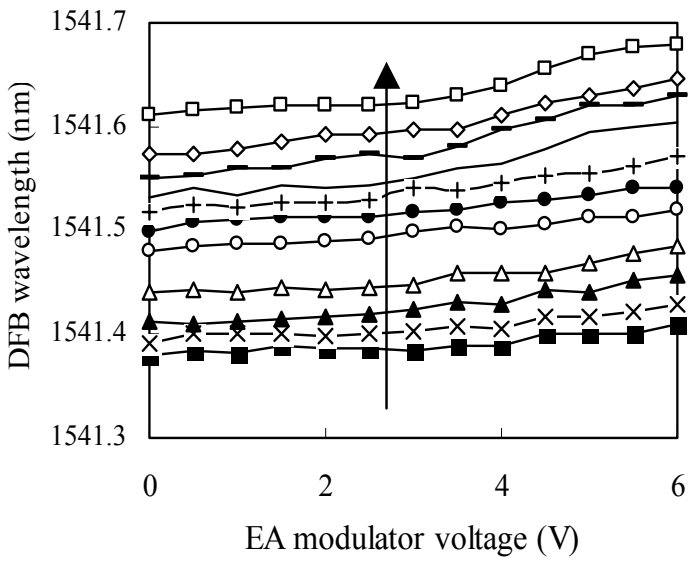

Fig. 2. DFB peak wavelength versus modulator voltage $\left(I_{\mathrm{LD}}=20 \mathrm{~mA}-75 \mathrm{~mA} ; 5 \mathrm{~mA}\right.$ step $)$

The length of the modulator and the DFB laser were 90-100 $\mu \mathrm{m}$ and $450 \mu \mathrm{m}$, respectively. The rear facet is high-reflection and the front anti-reflection coated. The peak wavelength is measured at the front facet with an optical spectrum analyzer.

\section{Measurement Results}

Figure 2 shows the DFB laser wavelength for different currents versus modulator voltage under continuous operation at room temperature. From Fig. 2, a positive wavelength shift was observed with increasing laser current and the modulator bias voltage, while small current operation resulted in small increase.

To examine the thermal issues, pulse operation was tried and the voltage dependence of the DFB wavelength was vanished. Increasing the EAM bias leads to a super linear rise of dissipated power in the EAM, heating up the modulator and resulting into the laser section. With these experimental results, we will discuss and simulate the thermal crosstalk phenomenon by a 3D finite method.

\section{References}

(1) M. Peschke, et al. : IEEE Photon. Technol. Lett., Vol.16, No.11, pp. 2508-2510 (2004)

(2) T. Takeuchi, et al. : IEEE Photon. Technol. Lett., Vol.9, No.5, pp.572-574 (1997) 


\title{
Thermal Crosstalk in Integrated DFB Laser-EA Modulators
}

\author{
Kayastha Madhusudan Non-member \\ Koichi Wakita Member \\ Kiyohide Baba Non-member
}

The wavelength red-shifting of integrated DFB (Distributed feedback) laser and EA (Electro-absorption) modulator with applied modulator bias voltage has been investigated and this phenomenon is estimated to be due to non-uniform temperature distribution in the device and caused by thermal crosstalk. From the experimental data and thermal analysis, increased absorbed photocurrents associated with EA modulator bias and dissipated power in the EA modulator lead to the temperature increase in continuously operating DFB laser, resulting wavelength red-shifting.

Keywords: integrated, DFB laser, thermal

\section{Introduction}

There is considerable interest in integrated DFB laser and EA modulator (EAM) as an attractive light source for high bit rate and long haul optical fiber communication systems because of their low driving voltage ${ }^{(1)}$, small size, compact structure, and low or even negative chirp characteristics ${ }^{(2)}$, and easy integration compared with others alternatives. However they show a red-shift of emission wavelength with an applied voltage for EAM. This leads to an adiabatic chirp parameter $\alpha_{H}$ upto 0.13 that is usually zero for decoupled devices like hybrid laser modulator integration $^{(3)}$.

The thermal crosstalk in integrated DFB laser and EA modulator operating at $1.3 \mu \mathrm{m}$ using InGaAs/InAlAs MQW structure has been reported in M. Peschke, et.al ${ }^{(3)}$. Although InGaAs/InAlAs MQW structure is superior to that of InGaAsP/InGaAsP MQW EA modulator because of its strong quantum confinement, the latter has been used practically because of its easy fabrication and the reported model has never applied for the later. In this paper we investigate the thermal crosstalk in integrated light source based on InGaAsP/InGaAsP MQW which has been more practically used. In general, the different sections of integrated device cannot be considered to be independent of each other. Due to the strong optical coupling between a DFB laser and an EA modulator, reflection from EA modulator can cause optical feedback. The electrical separation of the sections can be usually done by ion implantation, etched trenches or local zinc diffusion, but insufficient longitudinal resistances may lead to electrical crosstalk. This insufficient resistance causes non-uniform temperature distribution in the device. Therefore temperature changes in one section heat up the other section. The phenomenon in integrated DFB laser and EA modulator is called as "thermal crosstalk". In this paper we report the wavelength shift in integrated InGaAsP/InGaAsP MQW DFB laser and EA modulator as a function of injected current and analyze based on thermal crosstalk.

Chubu University

Department of Electrical and Electronics Engineering

1200, Matsumoto-cho, Kasugai-shi, Aichi-ken 487-8501

\section{Device Configuration}

Schematic of the contact layout of the integrated DFB laser and EA modulator is shown in Fig. 1. This device consists of DFB laser and EA modulator. The dashed line in Fig. 1 shows the separation between DFB laser and EA modulator. Length of the EA modulator and DFB laser were $90-100 \mu \mathrm{m}$ and $450 \mu \mathrm{m}$ respectively. The three dimensional view of integrated DFB laser and EA modulator device is schematically shown in Fig. 2.

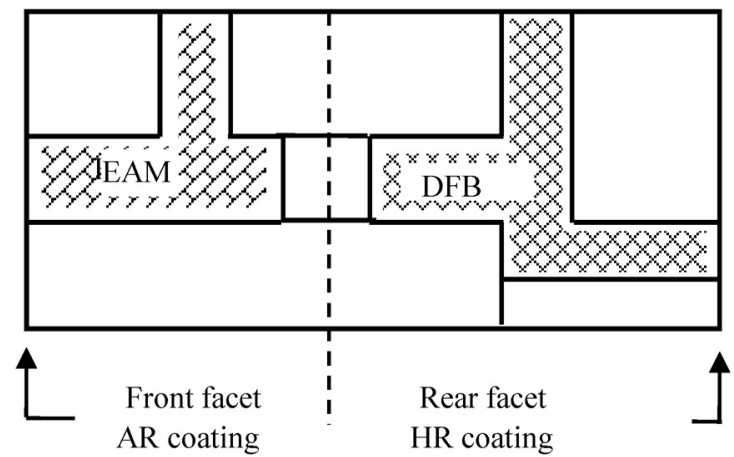

Fig. 1. Schematic of the contact layout of the device.

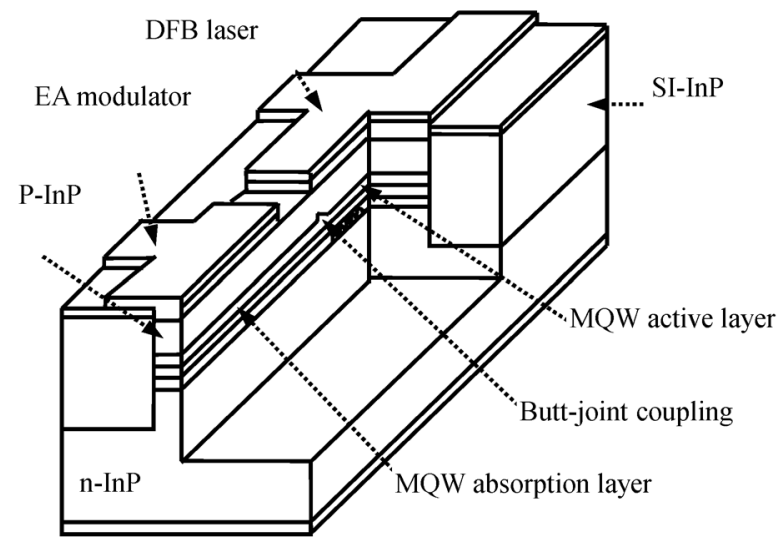

Fig. 2. Three dimensional view the integrated DFB laser and EA modulator. 
These two sections are integrated using butt-joint configuration on InP chip with high efficiency of $95 \%$. This butt-joint technique is commonly used for DFB-EAM fabrication because it enables us to optimize the characteristics of each MQW for the EAM and DFB laser with efficient optical transmission between them. The active area consists of an InGaAsP/InGaAsP multiple quantum well (MQW) structure on InP substrate. This is able to deliver high gain for forward current injection and high absorption swing for reverse voltage at $1541 \mathrm{~nm}$ operation wavelength. It consists of six pairs of strained InGaAsP MQW's as an active layer of a DFB laser by low-pressure metal organic vapor phase epitaxy.

The well is $6.7 \mathrm{~nm}$-thick InGaAsP with compressive strain and the barrier is $15.1 \mathrm{~nm}$-thick InGaAsP with a photoluminescence wavelength of $1.25 \mu \mathrm{m}$. This MQW active layer is etched off down to the substrate except in DFB laser region. Then, additional InGaAsP MQW's, with a well of $9.7 \mathrm{~nm}$ thick and barrier of $5 \mathrm{~nm}$ thick, is selectively grown as an absorption layer of EA modulator. The well is compressively strained by $0.5 \%$ and the barrier is tensilely strained to compensate the strain in the MQW layers. Two different contacts that are induced as a DFB laser and an EA modulator are aligned along a stripe waveguide buried in Fe-doped InP to reduce electrical capacitance and to make surface plane. The EA modulator buried with a S.I. (semi-insulating) InP is superior to high-mesa structures for the highly input optical power $^{(4)}$. The rear facet (DFB laser) is coated with high-reflection (HR) film whereas the front facet (EA modulator) is coated with anti-reflection film. The segment separation resistances were measured and were more than $30 \mathrm{k} \Omega$. The intensity modulated light is emitted at the front facet. This device can operate up to 40Gbps. The detail design and fabrication process of integrated DFB laser and EA modulator has been reported in Hiroaki Takeuchi, et. $\mathrm{al}^{(5)}$.

\section{Experimental Setup}

A schematic experimental block diagram for continuous wave (CW) operation is shown in Fig. 3. It consists of a PID instrument, a thermistor, and an optical spectrum analyzer (Ando AQ6317B)

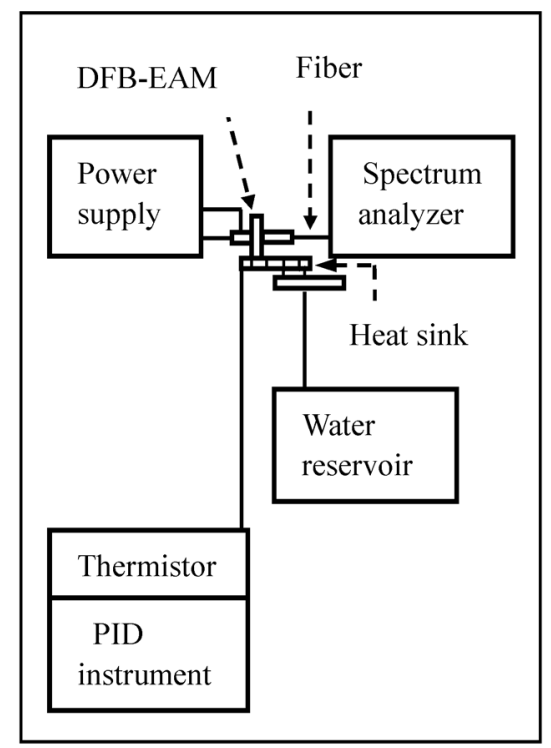

Fig. 3. Wavelength $(\lambda)$ and temperature rise measurement setup under continous wave operation. which operates Zerny and Turner type mechanism. DFB laser is forward biased whereas EA modulator is reversely biased. Here PID instrument is used for controlling the temperature of the integrated device, and an optical spectrum analyzer is used for taking the measurement of the wavelength as well as the power. Resolution of this optical spectrum analyzer is $0.01 \mathrm{~nm}$ but our measurement is taken at $25 \mathrm{~nm}$ span with a resolution of $0.2 \mathrm{~nm}$. The temperature sensitive device, a thermistor is used for the measurement of the temperature in the device with accurracy of $+/-0.1^{\circ} \mathrm{C}$. For this measurement process a Pertier cooler is also implemented. In order to take measurement, the output light of the integrated device is coupled with single mode optical fibre having semi-spherical edge and fed to optical spectrum analyzer. The wavelength shift under different values of currents or temperature in integrated device has been measured under both continous wave as well as pulse operation. For the pulse operation a series chip resistance of $50 \Omega$ was connected with EA modulator in order to match the impedence. The pulse width was $1 \mu$ s and the duty cycle was $1: 100$.

\section{Results and Discussion}

Figure 4 shows a typical wavelength spectrum of the optical output operating at the near threshold current. We can observe clear stopband associated with a DFB mode .

Wavelength is measured as a funtion of temperature under both continous wave and and pulse operation at lasing threshold current. It is shown in Figs. 5. and 6, respectively. Both measurement is taken as $0 \mathrm{~V}$ biased condition of EA modulator. From Figs. 5 and 6 , we calculate the value of $\Delta \lambda / \Delta T$ for both continuous operation as well as pulse operation. It has been determined that the value of the wavelength shift is greater in continuous operation as compared to pulse operation, $\Delta \lambda / \Delta T=$ $0.14 \mathrm{~nm} / \mathrm{deg}$ and $\Delta \lambda / \Delta T=0.12 \mathrm{~nm} / \mathrm{deg}$, respectively.

The emitting wavelength of the DFB laser as a function of reverse baised EA modulator voltages under a continous wave operation at room temperature is shown in Fig. 7. The measurement was taken at $27{ }^{\circ} \mathrm{C}$. The laser current was increased by $5 \mathrm{~mA}$ from $20 \mathrm{~mA}$ to $70 \mathrm{~mA}$ and the EA modulator reversed bias voltage was changed from $0 \mathrm{~V}$ to $-6 \mathrm{~V}$. From Fig. 7 , it is observed that the emitted DFB laser wavelength shifts to longer

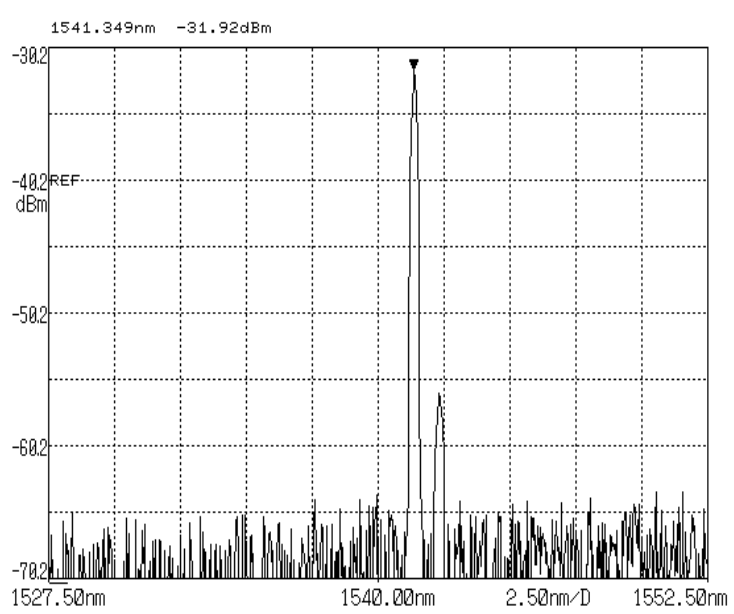

Fig. 4. Wavelength spectrum of the optical output operating near lasing thershold current. 


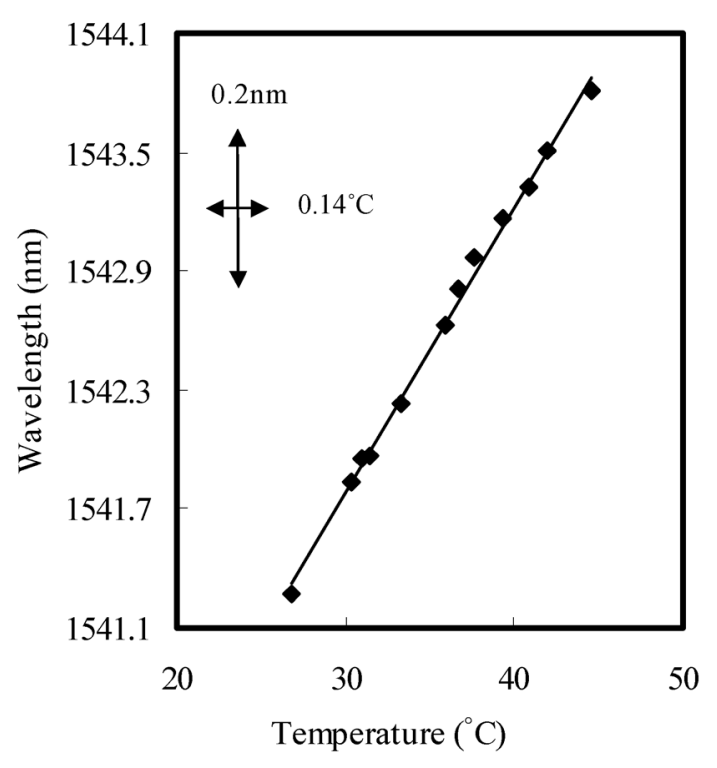

Fig. 5. Emitting wavelength $(\lambda)$ versus temperature $(T)$ for the continously operating DFB laser at lasing threshold current.

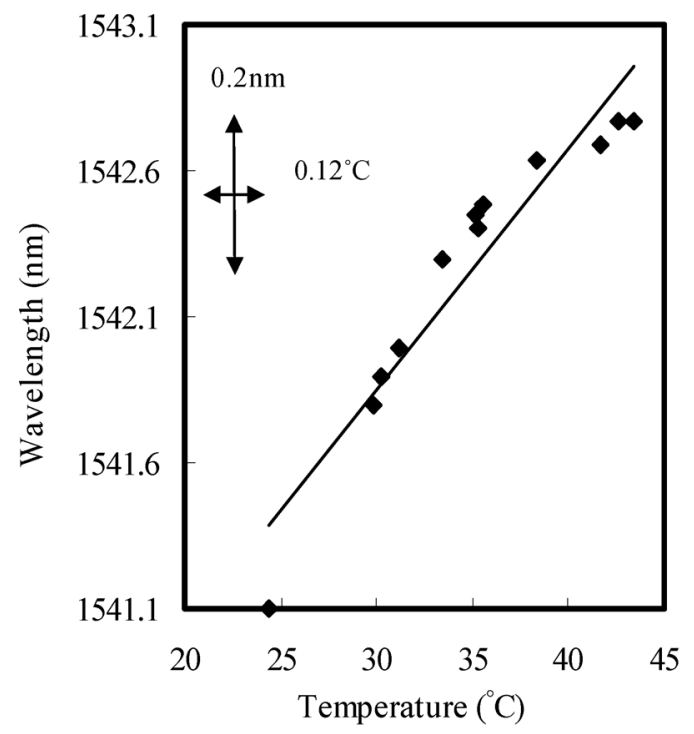

Fig. 6. Emitting wavelength $(\lambda)$ versus temperature $(T)$ for pulse operating DFB laser at lasing threshold current.

(red-shift) wavelength with increasing laser current and the modulator bias voltage, while small current operation resulted in small increase. In contrast, a red wavelength shift was observed exhibiting a steeper slope of $\lambda_{\mathrm{DFB}}\left(V_{\mathrm{EAM}}\right)$ with rising current and bias voltage.

To examine the thermal issues on integrated DFB laser and EA modulator, the measurement was repeated under pulse operation for the zero bias condition. The measurement result is shown in Fig. 8. Here the current dependence of the DFB wavelength has vanished under pulse operation, whereas large red-shift was observed under continuous wave operation. This indicates that wavelength shift is due to thermal effect. Actually the wavelength shift under the pulse current operation was compared with continuous operation at $0 \mathrm{~V}$ bias conditions. The wavelength shift is very small at pulse operation. This indicates that the DFB laser

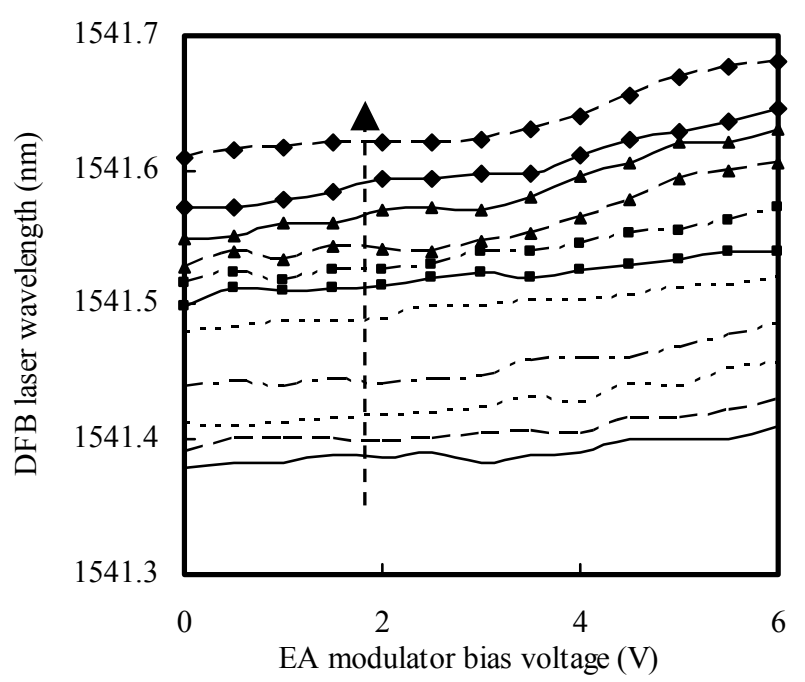

Fig. 7. DFB peak wavelength $(\lambda)$ versus modulator bias voltage as a junction of laser currents under continous wave operation at $27^{\circ} \mathrm{C}$. $\left(I_{\mathrm{DFB}}=20 \mathrm{~mA}\right.$ $70 \mathrm{~mA}$, step $5 \mathrm{~mA}$ ).

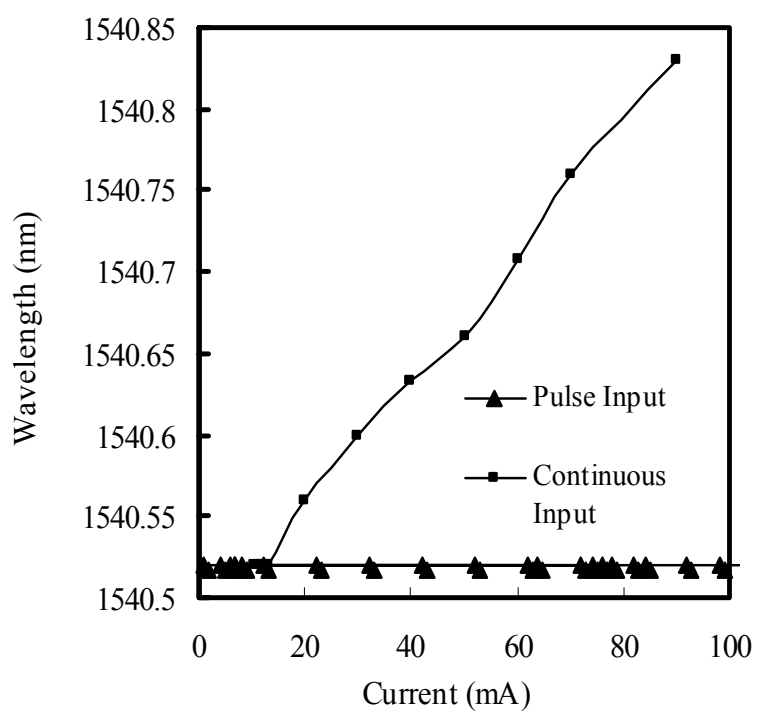

Fig. 8. Comparison of wavelength $(\lambda)$ under continuous wave and pulse operation at $20.6^{\circ} \mathrm{C}$. The EA modulator is under $0 \mathrm{~V}$ bias condition.

has less band-filling effects associated with injected current magnitude. Therefore the red-shift is due to self-heating of a DFB laser and thermal conduction from EA modulator that absorbed photocurrent.

Since incident light is attenuated along the modulator, the temperature distribution is not uniform. The integrated DFB laser and EA modulator will experience the strong thermal effect on both sections. Heat is generated in the active region of the modulator when a absorbed current, resulting from the absorption of light, flows perpendicularly to quantum wells. The heat generated is non-uniform along the length of device, since internal temperature depends on the operating conditions such as the applied voltage, absorbed current and optical power.

This variation of band-gap with temperature for InGaAsP/InP approximately obeys the empirical relation, first proposed by 
Varshni equations ${ }^{(6)}$.

$$
\operatorname{Eg}(T)=E g(0)-A T^{2} /(T+B) \cdots
$$

where $\operatorname{Eg}(T)$ and $\operatorname{Eg}(0)$ are the values of the energy gap at $\mathrm{T}^{\circ} \mathrm{K}$ and $0^{\circ} \mathrm{K}$, respectively. The parameters $A$ and $B$ are Varshni constants whose corresponding values are $0.582 \mathrm{meV} / \mathrm{K}$ and 316.67K respectively for our EAM device. These two values are calculated using parameters of binary elements, GaAs, InP, and $\operatorname{InAs}{ }^{(7)(8)}$.

There are mainly two sources of heating in the device. One is due to joule heating phenomenon and another is due to product of applied bias voltage and absorbed current.

Figure 9 shows photocurrent as a function of EA modulator bias voltage at different values of laser current. We can see that photocurrent is constant at the higher EA modulator bias voltage, but Fig. 7 shows a wavelength shift at the higher bias voltage. This wavelength shift is due to applied bias voltage. Though the absorbed current remains constant at higher bias voltage, the product of voltage and absorbed current still increases due to applied bias voltage and cause heating in the device. Therefore, temperature increases in the device due to heating effect causes shifting of wavelength.

Wavelength versus absorbed current as a function of laser curent under continuous wave operation at room temperature is shown in Fig. 10. We observe that wavelength shift occurs at constant absorbed current. Figure 9 shows that saturation of absorbed current occurs at bias voltage greater than $3 \mathrm{~V}$. But from Fig. 7, wavelength shift with increasing laser current and the modulator bias voltage is observed. Therefore, this wavelength shift at constant absorbed current is due to applied reverse bias voltage in EA modulator.

The saturation of absorbed current is investigated using the extinction ratio for EA modulator as shown in Fig.11. The extinction ratio is at most $8.8 \mathrm{~dB}$ and extinction ratio does not increase so much over bias voltage of $3 \mathrm{~V}$. This indicates the absorption coefficient does not increase due to short EAM length and poor optical confinement ${ }^{(5)}$ and low extinction ratio.

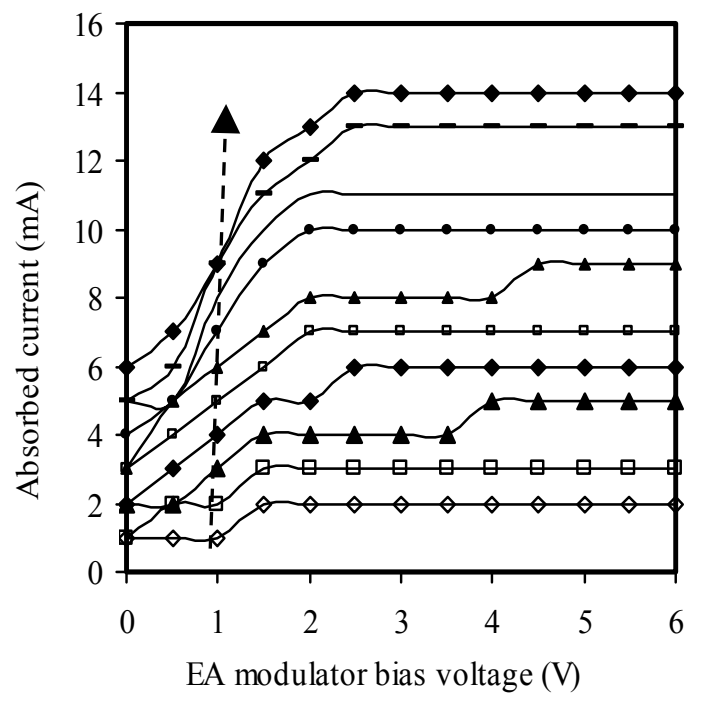

Fig. 9. Absorbed current versus EA modulator bias voltage as a function of laser current under continous wave operation at $27^{\circ} \mathrm{C}$. $\left(I_{\mathrm{DFB}}=20 \mathrm{~mA}-65 \mathrm{~mA}\right.$, step $5 \mathrm{~mA})$.
The thermal crosstalk phenomenon was simulated by using the commercially software MATLAB. The length of the DFB laser and EA modulator was assumed as of the actual device. The standard heat transport equation is given as ${ }^{(3)}$,

$$
\nabla(k \nabla T)=Q_{i}
$$

Where, subscript $i$ indicates laser and modulator regions, $k$ is the thermal conductivity $(68 \mathrm{~W} / \mathrm{Km}$ for $\mathrm{InP})$ and $T$ is temperature $(K)$.

The dissipated heat power density $\left(Q_{i}\right)$ are given by

$$
\begin{aligned}
& Q_{\mathrm{DFB}}=\frac{1}{d_{\mathrm{PN}}}\left(V_{\mathrm{DFB}} J_{\mathrm{DFB}}-\frac{P_{\mathrm{DFB}}}{w L_{\mathrm{DFB}}}\right) \\
& Q_{\mathrm{EAM}}=\frac{1}{d_{\mathrm{PN}}}\left(V_{\mathrm{EAM}}+\frac{h \omega}{e}\right) J_{\mathrm{EAM}}(x)
\end{aligned}
$$

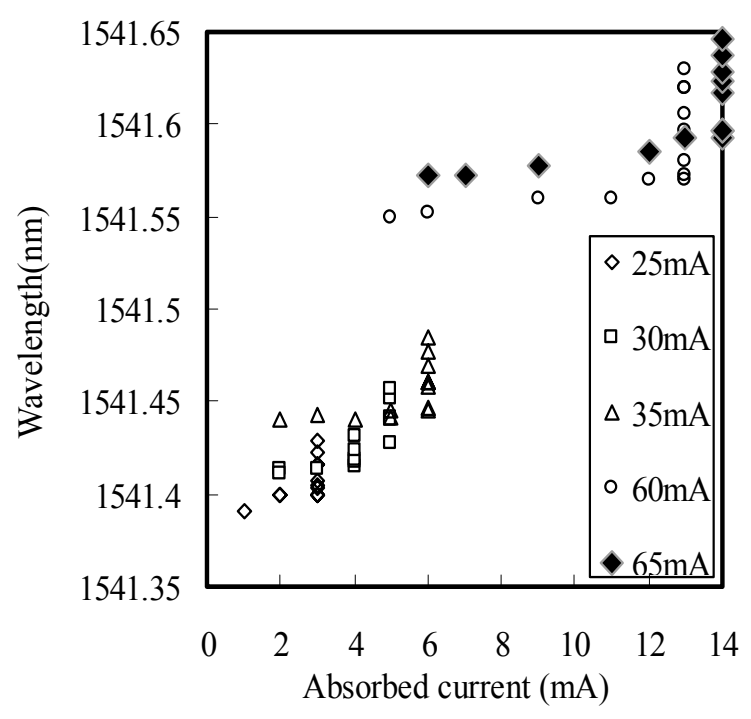

Fig. 10. Wavelength versus absorbed current as a function of laser current under continous wave operation at $27^{\circ} \mathrm{C}$.

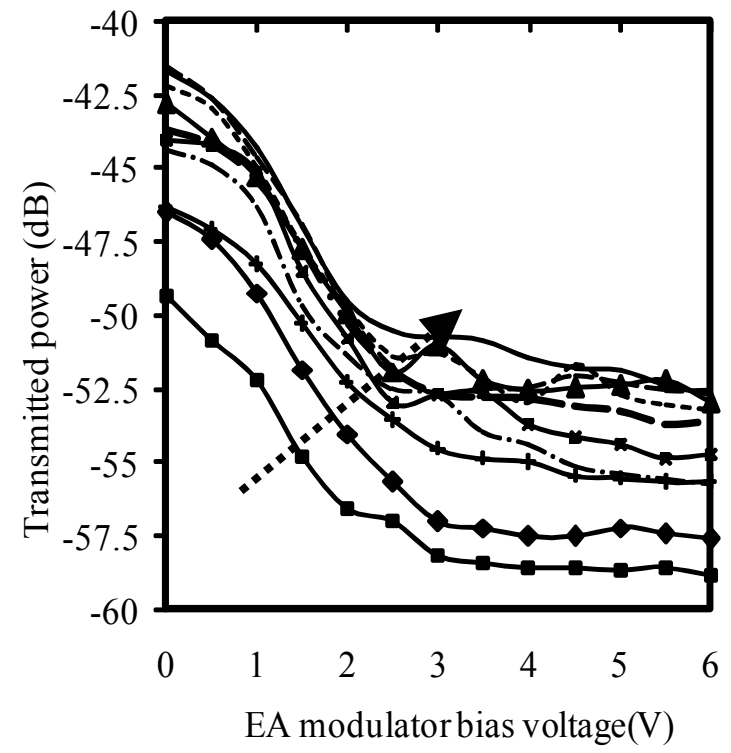

Fig. 11. Transmitted power as a function of EA modulator bias voltage $\left(I_{\mathrm{DFB}}=20 \mathrm{~mA}-65 \mathrm{~mA}\right.$, step $\left.5 \mathrm{~mA}\right)$. 
Where $V_{i}$ are the voltages across the intrinsic region,

$d_{\mathrm{PN}}$ is the intrinsic region thickness,

$J_{i}$ are the corresponding current densities,

$P_{\mathrm{DFB}}$ is the total emitted light from the laser diode,

$w$ is the width of the active region,

$\hbar$ is reduced Planck's constant

$L_{\mathrm{DFB}}$ is length of laser.

For the sake of simplicity, all light generated in the laser is assumed to couple into EA modulator. The current density in the laser $J_{\mathrm{DFB}}$ is assumed to be constant, while the current density in the EA modulator section $J_{\text {EAM }}$ reduces exponentially with the light intensity with the voltage dependent absorption coefficient $\alpha$.

$$
J_{\mathrm{EAM}}(x)=\frac{e}{\hbar} \frac{\alpha}{\omega} \frac{P_{\mathrm{DFB}}}{w} \exp (\alpha x)
$$

Using the above equation and parameters, the temperature distribution in integrated DFB laser and EA modulator was estimated. We have assumed the active region to be $4 \mu \mathrm{m}$ wide and $300 \mathrm{~nm}$ thick. Parameters for the characteristics equations are the threshold current $I_{t h}=12 \mathrm{~mA}$, the differential quantum efficiency at DFB-EAM interaction $\eta_{d}=0.35 \mathrm{~W} / \mathrm{A}$, the kink voltage $V_{0}=$ $0.80 \mathrm{~V}$, the laser series resistance $R_{S}=10 \Omega$, and the thermal wavelength shift $\Delta \lambda / \Delta T=0.1 \mathrm{~nm} / \mathrm{K}$. They were taken from the measurement data of the integrated DFB laser-EA modulator.

Figure 12 shows the temperature distribution along the active area for different value of absorption coefficient $(300,200$ and 100 $/ \mathrm{cm})$ at the total emitted light from the laser diode $\left(P_{\mathrm{DFB}}\right)$ as $12 \mathrm{~mW}$. This shows that temperature increase is higher at higher value of the absorption coefficient and temperature rise takes place in the initial $20 \mu \mathrm{m}$ section of the EA modulator. This shows that temperature rise is higher at EA modulator section of the device. Here the peak temperature rise is not located at the interface of the two section because at the EA modulator side the absorbed current will be maxmimum and resulting dissipation power will be maximum, however rising heat conducts to lower temperature side (DFB laser section) and the temperature peak position will shift into the EA modulator side. The decaying curve at the DFB laser section indicates a transfer of heat from EA modulator section

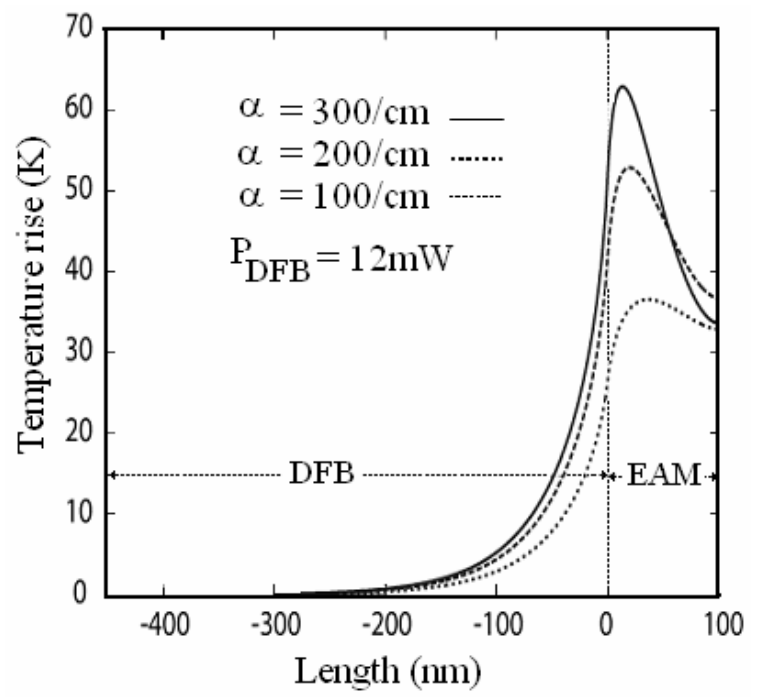

Fig. 12. Simulated temperature distribution along the active area of integrated DFB laser and EA modulator. toward DFB laser section since temperature rise is higher at EA modulator section. However simulated temperaure rise value is higher compared with practical result. This discrepancy is attributed to the poor optical coupling efficiency and poor estimation of thermal crosstalk between chips and chips and non ideal thermal coupling between the chips to the heat sink and the non-self-consistent nature of the simulation. From the extinction data in Fig. 11, we can estimate the absorption coeffficient change to be at most $203 / \mathrm{cm}$. This value is little bit smaller compared with those of Fig. 12.

\section{Conclusion}

Wavelength shift with the applied modulator bias has been investigated. Increasing the EA modulator bias leads to rise of dissipation power in EA modulator section, heating up the EA modulator and reached into laser section. Wavelength shift vanish in pulse operation has been investigated. By measurement corresponding simulation, the phenomenon was identified to be the caused by thermal coupling between the sections.

(Manuscript received Mar. 16, 2007, revised Aug. 30, 2007)

\section{References}

(1) K. Wakita and I. Kotaka : "Multiple-quantum-well optical modulators and their monolithic integration with DFB lasers for optical fiber communications", Microwave and optical Technol. Lett., Vol.7, No.3, pp.120-128 (1994)

(2) R. Salvatore, R. Sahara, M. Bock, and I. Libenzon : "Electroabsorption modulated laser for long transmission spans", IEEE J. Quantum Electronic Vol.38, No.5, pp.464-476 (2002)

(3) M. Peschke, P. Gerlach, B. K. Saravanan, and B. Stegmueller : "Thermal Crosstalk in integrated laser-modulators", IEEE Photon. Technol. Lett., Vol.16, No.11, pp.2508-2510 (2004)

(4) K. Wakita, I. Kotaka, S. Matsumoto, R. Iga, S. Kondo, and Y. Noguchi : "Very-high-allowability of incidental optical power for polarizationinsensitive InGaAs/InAlAs multiple quantum well modulators buried in semi-insulating InP", Jpn J.Appl. Physics, Vol.37, No.3B, pp.1432-1435 (1998)

(5) H. Takeuchi, K. Tsuzuki, K. Sato, M. Yamamoto, Y. Itaya, A. Sano, M. Yoneyama, and T. Otsuji : "Very high-speed light-source module up to $40 \mathrm{~Gb} / \mathrm{s}$ containg an MQW electroabsorption modulator integrated with a DFB laser", IEEE J. Sel. Top. Quantum Electronics, Vol.3, No.2, pp.336-343 (1997)

(6) P. Bhattacharya : "Semiconductor optoelectronic devices", Prentice-Hall of India private limited (1995)

(7) M. Peschke : "Thesis on laser diodes integrated with electroabsorption modulators for $40 \mathrm{~Gb} / \mathrm{s}$ datatransmission".

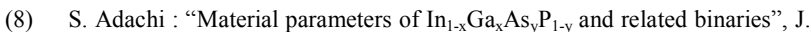
Appl. Phys, Vol.53, No.12, pp.8775-8793 (1982)

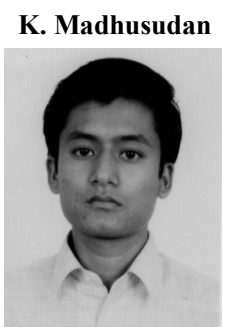

(Non-member) was born in Jan, 1980. He received B.E degree in Electronics and Communication Engineering from Pokhara Engineering College, Pokhara, Nepal, in 2004AD. Now he is a M.E student in the Department Electronics and Information Engineering of Chubu University, Aichi Japan. His research interests are concerned with semiconductor lasers and multiple quantum well optical modulators for optical fiber communication. He is student member of IEEE (Institute of Electrical and Electronics Engineers), JSAP (Japan Society of Applied Physics) and NEA (Nepal Engineer Association). 
Koichi Wakita (Member) received the B.S degree in Electronics

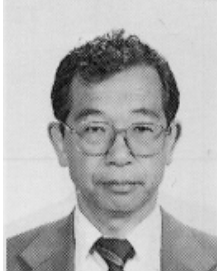

Engineering from the Nagoya Institute of Technology, in 1968 and the M.S. and Dr.Eng. degrees from the Nagoya University, in 1970 and 1976, respectively. In 1973, he joined NTT Electrical Communication Laboratories, Japan. He had been engaged in research and development on semiconductor lasers and multiple quantum well optical modulators for optical fiber communication. In 1997, he joined the Department of Electronics and Information Engineering of the Chubu University, Aichi, Japan, as a professor. He is a member of the IEICE of Japan, the Insistute of Electrical Engineers of Japan, and Japan Society of Applied Physics.
Kiyohide Baba (Non-member) was born on Nov 3, 1942 in Ina,

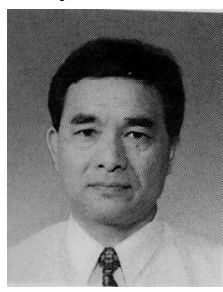

Japan. He received the B.S degree in Electronics Engineering from the Nagoya Institute of Technology, in 1966. In 1996, he joined the Department of Electronics and Information Engineering of the Chubu University, Aichi, Japan, as a professor. His research interests are concerned with ionospheric propagation of VLF radio wave and numerical methods to wave propagation He received the Doctor of Engineering in 1992. He is a member of the IEICE of Japan, the Institute of Electrical Engineers of Japan, and Japan Society of Applied Physics. 